\title{
Relativistic time-dependent quantum dynamics across supercritical barriers for Klein-Gordon and Dirac particles
}

\author{
M. Alkhateeb $\odot,{ }^{1}$ X. Gutiérrez de la Cal, ${ }^{2}$ M. Pons $\odot,{ }^{3}$ D. Sokolovski, ${ }^{2,4}$ and A. Matzkin ${ }^{1}$ \\ ${ }^{1}$ Laboratoire de Physique Théorique et Modélisation, CNRS Unité 8089, CY Cergy Paris Université, 95302 Cergy-Pontoise cedex, France \\ ${ }^{2}$ Departmento de Química-Física, Universidad del País Vasco, UPV/EHU, 48940 Leioa, Spain \\ ${ }^{3}$ Departamento de Física Aplicada, Universidad del País Vasco, 48013 Bilbao, Spain \\ ${ }^{4}$ IKERBASQUE, Basque Foundation for Science, E-48011 Bilbao, Spain
}

(Received 14 December 2020; revised 18 March 2021; accepted 18 March 2021; published 5 April 2021)

\begin{abstract}
We investigate wave-packet dynamics across supercritical barriers for the Klein-Gordon and Dirac equations. Our treatment is based on a multiple scattering expansion (MSE). For spin-0 particles, the MSE diverges, rendering invalid the use of the usual connection formulas for the scattering basis functions. In a time-dependent formulation, the divergent character of the MSE naturally accounts for charge creation at the barrier boundaries. In the Dirac case, the MSE converges and no charge is created. We show that this time-dependent charge behavior dynamics can adequately explain the Klein paradox in a first-quantized setting. We further compare our semianalytical wave-packet approach to exact finite-difference solutions of the relativistic wave equations.
\end{abstract}

DOI: 10.1103/PhysRevA.103.042203

\section{INTRODUCTION}

One of the salient features of relativistic quantum mechanics is the intrinsic mixture of particles with their corresponding antiparticles. This aspect already appears in "first-quantized" single-particle relativistic quantum mechanics (RQM) [1]. This phenomenon becomes prominent when a particle is placed in a very strong external classical field, a field strong enough to close the gap between particles and antiparticles-a supercritical field.

Most textbooks, as well as the overwhelming majority of past works employ time-independent stationary phase or plane-wave arguments when dealing with supercritical fields within RQM. As is well known, in quantum scattering theory time-independent quantities such as cross-sections or energy levels can be readily computed, but it is often ambiguous to attempt to infer the dynamics from considerations involving a single plane wave. This is even more the case when relativistic phenomena are investigated: One has to deal with additional difficulties, such as the breakdown of the usual connection formulas linking the scattering solutions in different regions, or the Klein paradox - a phenomenon usually expressed as a current reflected from a supercritical step or barrier higher than the incident one. Standard textbooks (e.g., [1-4]) give different, often conflicting accounts of the Klein paradox. This situation is reflected even in recent works [5-15], that reach different conclusions generally based on time-independent considerations.

In part for this reason, it is often stated, in different situations dealing with the Klein paradox, that a RQM approach is inadequate and that a quantum field theory (QFT) treatment is necessary (see, e.g., [3] for the step case, or [8] for the barrier case). Nevertheless even in a first-quantized framework the RQM wave equations have charge creation built in. We argue in this paper that this aspect is best understood by considering the time-dependent wave-function dynamics. We will assess whether this leads to a consistent first-quantized explanation of the Klein paradox. Interestingly, other very recent works have focused on different time-dependent aspects of the relativistic wave equations $[16,17]$.

In this paper we will develop a time-dependent wavepacket treatment suited to investigate the dynamics of spin- 0 bosons and spin- $1 / 2$ fermions in model supercriticial barriers. In order to develop our semianalytic wave-packet approach, we will rely on a multiple scattering expansion (MSE). We will see that the nature of the MSE is different for solutions of the Klein-Gordon and Dirac equations. In the Klein-Gordon case, the MSE diverges, physically corresponding to charge creation as the wave packet hits a barrier's edge. This implies that the usual connection formulas between wave functions in different regions, which are obeyed when a single step potential is considered, are not applicable when dealing with supercritical barriers. Employing connection formulas leads to inconsistencies, such as superluminal traversal times, that were recently noted [8] though (incorrectly, as we will see) attributed to the limitations of the first-quantized formalism. In the Dirac case, the MSE converges, as no charge is created when the wave packet hits the barrier. This leads to an interpretation of Klein tunneling that is qualitatively different from the bosonic case. This also ensures that the connection formulas, which have been employed in a countless number of works, remain valid.

The multiple scattering expansion will be given in Sec. II, after introducing the model barriers we will be working with. These ingredients will be employed in Sec. III to build wave packets. We will then give a couple of examples displaying the dynamics of a bosonic or fermionic particle impinging on a supercritical barrier. Our wave-packet results will be 


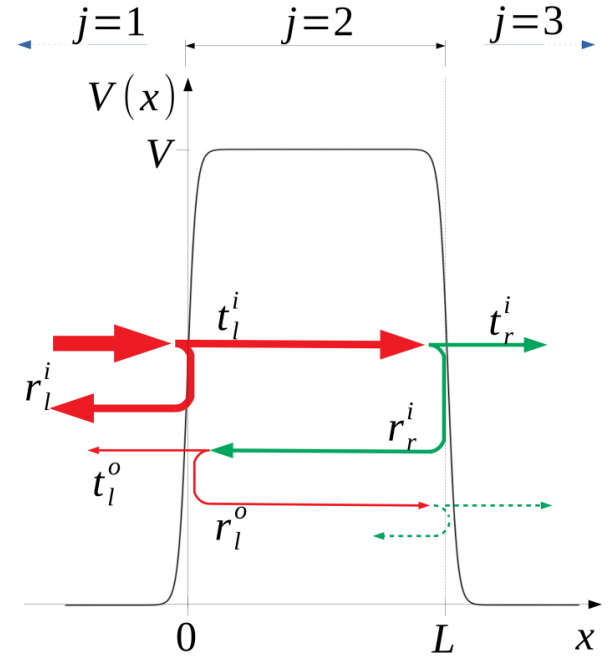

FIG. 1. A generic barrier is shown along with the three regions $j=1,2,3$. The scattering amplitudes of the multiple scattering expansion at the left $(l)$ and right $(r)$ edges are indicated for an incoming wave boundary condition (see text for details).

compared to numerical solutions obtained from a code we have developed to solve numerically the relativistic wave equations. Our results will be discussed in Sec. IV. We will more specifically focus on the extent to which the Klein paradox can be accounted for within a first-quantized framework. We close the paper with a Conclusion.

\section{CHARGE CREATION AND MULTIPLE SCATTERING EXPANSIONS}

\section{A. Potential barriers}

We are interested in this work by the dynamics of a relativistic "particle" impinging on a one-dimensional static barrier of width $L$. The barrier should be discriminated from the step, which is by far the case that has most often been considered in studies of the Klein paradox. The relevant wave equations for spin-0 and spin-1/2 particles are recalled in Sec. S1 of the Supplemental Material [18].

The simplest case is the rectangular barrier defined by $V(x)=V \theta(x) \theta(L-x)$ where $\theta$ denotes the unit-step function and $V$ denotes the barrier height. We will also consider smooth barriers, for which we will employ the potential $V_{s}(x, \epsilon)=$ $\frac{V}{2}\{\tanh (\epsilon x)-\tanh [\epsilon(x-L)]\}$ since for this potential analytical solutions are known [19]; $\epsilon$ is the smoothness parameter.

\section{Subcritical barriers}

Let us consider a rectangular barrier. Plane-wave solutions of the canonical Klein-Gordon (KG) equation (see [18]) are of the form

$$
\psi_{j}^{ \pm}(t, x)=\left(A_{j}^{ \pm} e^{i p_{j} x / \hbar}+B_{j}^{ \pm} e^{-i p_{j} x / \hbar}\right) e^{\mp i E\left(p_{1}\right) t / \hbar},
$$

where $j=1,2,3$ denotes the regions depicted in Fig. 1 and the \pm signs correspond to states with positive and negative energies $\pm E\left(p_{1}\right)$ with $E\left(p_{1}\right)=\sqrt{m^{2} c^{4}+p_{1}^{2} c^{2}} \cdot p_{j}$ is the momentum; for positive energies, $p_{j}>0$ gives a wave moving from left to right (but from right to left for negative energies).

As is well known [1,20], for "subcritical" potentials ( $p_{2}$ is imaginary) plane-waves scattering is similar to the usual nonrelativistic situation (small transmission amplitude and exponentially decreasing waves). Assume boundary conditions for which an incident positive energy wave travels from left to right; this imposes $B_{3}=0$ and for definiteness we set the incoming amplitude to $A_{1}=1$. The other amplitudes $A_{j}$ and $B_{j}$ are deduced by matching the wave functions and their space derivatives at the boundaries $x=0$ and $x=L$ (for reasons that will become clear below, we will not need to deal with boundary conditions for negative energy plane waves; we henceforth write $A$ for $A^{+}$, etc.). This way of obtaining the amplitudes does not necessarily hold when $V$ becomes supercritical.

\section{Supercritical barriers}

A supercritical potential is a potential high enough to give rise to Klein tunneling [21], whereby the incoming wave packet penetrates undamped ( $p_{2}$ is real) inside the barrier. In the bosonic case, this gives rise [20] to superradiance (a reflected current higher than the incoming one). In the fermionic case there is no superradiance (although some authors suggest differently; see, e.g., [2,13]), and supercritical steps have been deemed to have an acceptable interpretation only within a QFT approach [22-24], a point that appears to be supported by the wide variety of conflicting interpretations of Klein tunneling that have been proposed within the first-quantized framework [5-15].

\section{B. Multiple scattering expansions}

It is well known that when transmission of waves across several media takes place, one has to take into account a multiple scattering process. Referring again to Fig. 1, consider an asymptotically free (at $x=-\infty$ ) wave coming toward the barrier (we set again $A_{1}=1$ and $B_{3}=0$ ). Reflection of the incoming wave on the barrier takes place with amplitude $r_{l}^{i}$, which is the reflection amplitude of a step. The transmitted amplitude at that point, $t_{l}^{i}$, is that of a step, but the wave traveling inside the barrier reaches the right edge and gets transmitted and reflected with amplitudes $t_{r}^{i}$ and $r_{r}^{i}$. This reflected wave travels back towards the left side of the barrier, getting reflected and transmitted with coefficients $r_{l}^{o}$ and $t_{l}^{o}$. This process is iterated an infinite number of times yielding

$$
\begin{array}{cc}
r \equiv B_{1}=r_{l}^{i}+t_{l}^{i} t_{l}^{o} r_{r}^{i} \sum_{n \geqslant 0}\left(r_{l}^{o} r_{r}^{i}\right)^{n}, & t \equiv A_{3}=\sum_{n \geqslant 0} t_{l}^{i}\left(r_{l}^{o} r_{r}^{i}\right)^{n} t_{r}^{i} \\
A_{2}=\sum_{n \geqslant 0} t_{l}^{i}\left(r_{l}^{i} r_{l}^{o}\right)^{n}, & B_{2}=\sum_{n \geqslant 0} t_{l}^{i} r_{r}^{i}\left(r_{l}^{o} r_{r}^{i}\right)^{n} \\
A_{1}=1, & B_{3}=0 .
\end{array}
$$


The amplitudes obtained by using this MSE should match those obtained by employing the usual connection formulas at the boundaries, but this will happen only provided the sums in Eq. (2) converge.

\section{Klein-Gordon equation: Divergent multiple scattering expansion}

Let us consider the $\mathrm{KG}$ equation in the presence of a supercritical step $V \theta(x)$ at $x=0$. Scattering of a positive energy plane wave coming from the left, as given by Eq. (1), sets $\bar{A}_{1}=1, \bar{B}_{2}=0$ (we use the bar to avoid confusion between the step and barrier amplitudes). $\bar{A}_{2}$ and $\bar{B}_{1}$ are thus obtained by applying the boundary conditions at $x=0$, yielding $\bar{B}_{1}=$ $\left(p_{1}-p_{2}\right) /\left(p_{1}+p_{2}\right)$ and $\bar{A}_{2}=2 p_{1} /\left(p_{1}+p_{2}\right)$ where following our notation given above, $p_{1}>0$ is the momentum of the incoming plane wave (in region 1) and

$$
p_{2}\left(p_{1}\right)=-\frac{1}{c} \sqrt{p_{1}^{2} c^{2}-2 V\left(m^{2} c^{4}+p_{1}^{2} c^{2}\right)^{1 / 2}+V^{2}} .
$$

The amplitudes $\bar{B}_{1}$ and $\bar{A}_{2}$ of the step correspond to the amplitudes $r_{l}^{i}$ and $t_{l}^{i}$ of the barrier MSE, Eq. (2). $r_{r}^{i}$ and $t_{r}^{i}$ are obtained by considering the step $V \theta(L-x)$, and $r_{l}^{o}$ and $t_{l}^{o}$ arise from the step $V \theta(x)$ with a wave coming from the right (see Sec. S2 of [18]). By inserting the values for these elementary scattering amplitudes into the MSE of Eq. (2), we obtain the scattering amplitudes for the rectangular barrier. These amplitudes converge if $\left|\left(p_{1}-p_{2}\right) /\left(p_{1}+p_{2}\right)\right|<1$ in which case it can be checked that the amplitudes $A_{j}$ and $B_{j}$ match the ones obtained by using the connection formulas at the boundaries.

This condition is fulfilled for subcritical barriers (both $p_{1}$ and $p_{2}$ are positive) but for supercritical barriers, we have $p_{1}>0$ and $p_{2}<0$ and the MSE diverges. This divergence does not make much sense in a stationary plane-wave picture, in which the scattering amplitudes become infinite, but we will see below in Sec. III that in a time-dependent approach, the divergence corresponds physically to the creation of charge each time a wave packet hits a barrier edge.

Interestingly, if the "converged" amplitudes (usually obtained by employing the connection formulas) are employed in the supercritical case, unphysical results are obtained. It was recently noticed [8] in a plane-wave analysis that the barrier traversal time defined from the phase energy derivative was superluminal in the supercritical case, an unphysical result attributed to the limitations of the first-quantized formalism. In a wave-packet approach, building wave packets with the converged amplitudes results in an acausal wave packet coming out from the right side of the supercritical barrier before the incoming wave packet has even hit the barrier [25]. Other works have also employed the connection formulas in a supercritical context $[6,12]$.

\section{Dirac equation: Convergent multiple scattering expansion}

The derivation of the MSE for the Dirac equation is similar to the one we have given for the KG equation. The plane-wave solutions of the two-component state $\Phi(t, x)$ take the form

$$
\Phi_{j}^{ \pm}(t, x)=\left[A_{j}^{ \pm}\left(\begin{array}{c}
1 \\
\alpha_{j}^{ \pm}\left(p_{1}\right)
\end{array}\right) e^{i p_{j} x / \hbar}+B_{j}^{ \pm}\left(\begin{array}{c}
1 \\
-\alpha_{j}^{ \pm}\left(p_{1}\right)
\end{array}\right) e^{-i p_{j} x / \hbar}\right] e^{-i \sqrt{m^{2} c^{4}+p_{1}^{2} c^{2}} t / \hbar} n_{j}^{ \pm}\left(p_{1}\right)
$$

where the coefficients $\alpha_{j}^{ \pm}$are given by Eqs. (S-20)-(S-23) of [18]. $j$ refers again to the three regions depicted in Fig. 1 and $n_{j}^{ \pm}$are normalization coefficients.

As in the KG case, let us treat the barrier as a multiple scattering expansion, with the same boundary conditions. The amplitudes $A_{j}^{ \pm}$and $B_{j}^{ \pm}$are again given by Eq. (2), with coefficients $r_{l}^{i}, t_{l}^{i} \ldots$ that are different for positive and negative energy wave functions. As in the KG case, the MSE for a rectangular barrier is built from the reflection and transmission amplitudes $\bar{A}_{j}^{ \pm}$and $\bar{B}_{j}^{ \pm}$of the steps $V \theta(x)$ and $V \theta(L-x)$. The matching condition $\Phi_{1}^{+}(t, x=0)=\Phi_{2}^{+}(t, x=0)$ yields

$$
\bar{B}_{1}^{+}=\frac{\alpha_{1}^{+}\left(p_{1}\right)-\alpha_{2}^{+}\left(p_{1}\right)}{\alpha_{1}^{+}\left(p_{1}\right)+\alpha_{2}^{+}\left(p_{1}\right)}, \quad \bar{A}_{2}^{+}=\frac{2 \alpha_{1}^{+}\left(p_{1}\right)}{\alpha_{1}^{+}\left(p_{1}\right)+\alpha_{2}^{+}\left(p_{1}\right)} \frac{n_{1}^{+}}{n_{2}^{+}} .
$$

The amplitudes $\bar{B}_{1}$ and $\bar{A}_{2}$ of the step correspond to the amplitudes $r_{l}^{i+}$ and $t_{l}^{i+}$ entering the MSE for the barrier. The other step amplitudes are obtained in the same manner [see Eq. (S-15) of [18]]. It can be seen that the series converge provided $\left|\frac{\alpha_{1}^{+}\left(p_{1}\right)-\alpha_{2}^{+}\left(p_{1}\right)}{\alpha_{1}^{+}\left(p_{1}\right)+\alpha_{2}^{+}\left(p_{1}\right)}\right|<1$ which is verified since $\alpha_{2}^{+}\left(p_{1}\right)$ is positive when $V$ is supercritical.

Therefore for the Dirac equation, the MSE converges. The usual connection formulas at $x=0$ and $x=L$ may be em- ployed to obtain directly the barrier amplitudes $A_{j}^{ \pm}$and $B_{j}^{ \pm}$ given by Eqs. (S-24)-(S-27) of [18]. Note that most past works (e.g., [12,26,27]) have indeed employed such connection formulas without, however, examining the justifications for their use.

\section{WAVE-PACKET DYNAMICS}

\section{A. Construction from plane-wave expansions}

The most straightforward way to construct wave packets starting from an initial distribution is to employ a plane-wave expansion, valid everywhere except in the slope region for a sufficiently steep barrier.

\section{Klein-Gordon equation wave packets}

The Klein-Gordon plane waves were given in Eq. (1). These solutions can be expressed in Hamiltonian form [18], as

$$
\begin{aligned}
\Psi_{j}^{ \pm}(t, x)= & N\left(\begin{array}{l}
m c^{2} \pm \sqrt{m^{2} c^{4}+p_{j}^{2} c^{2}} \\
m c^{2} \mp \sqrt{m^{2} c^{4}+p_{j}^{2} c^{2}}
\end{array}\right)\left(A_{j}^{ \pm} e^{i p_{j} x / \hbar}\right. \\
& \left.+B_{j}^{ \pm} e^{-i p_{j} x / \hbar}\right) e^{\mp i \sqrt{m^{2} c^{4}+p_{1}^{2} c^{2}} t / \hbar}
\end{aligned}
$$


where $p_{3}=p_{1}$ and $p_{2}$ is given by Eq. (3), the amplitudes $A_{j}^{ \pm}$ and $B_{j}^{ \pm}$are given by Eq. (2), and $N$ is a global normalization constant.

Assume that at $t=0$ we have an initial wave function $G(t=0, x)=\left(\varphi_{G}(0, x), \chi_{G}(0, x)\right)$ in region 1 , to the left of the barrier. The time evolution can be employed by applying the pseudounitary evolution operator on $G(t=0, x)$, or equivalently by using the Fourier transform $G(t=0, x)=$ $\int d p e^{i p x / \hbar} \hat{G}(t=0, p)$. The time-evolved wave packet can then be written as

$$
\begin{aligned}
G(t, x)= & \sum_{j} \theta_{j} \int d p_{1}\left[c_{K G}^{+}\left(p_{1}\right) \Psi_{j}^{+}\left(t, x ; p_{1}\right)\right. \\
& \left.+c_{\mathrm{KG}}^{-}\left(p_{1}\right) \Psi_{j}^{-}\left(t, x ; p_{1}\right)\right],
\end{aligned}
$$

where $\theta_{j}$ ensures each expression is used only in the region $j$ in which it is valid, as per Fig. 1 [explicitly, $\theta_{1}=\theta(-x), \theta_{2}=$ $\theta(x) \theta(L-x)$, and $\left.\theta_{3}=\theta(x-L)\right]$.

To be specific, we will choose an initially localized state of the form

$$
G(0, x)=\left(\exp \left(\frac{-\left(x-x_{0}\right)^{2}}{4 d^{2}}-i p_{0} x / \hbar\right), 0\right) .
$$

Picking $x_{0}$ far to the left of the barrier and $p_{0}>0$, the choice (8) gives an initial state with positive charge. The coefficients $c_{\mathrm{KG}}^{ \pm}=\left\langle\Psi_{1}^{ \pm} \mid G\right\rangle_{\mathrm{KG}}$ are readily computed [Eq. (S-19) of [18]] and it can be seen that $c_{\mathrm{KG}}^{-}$is nonzero (although it is small for nonrelativistic velocities). This negative energy component moves to the left (recall that $p_{0}>0$ yields an antiparticle that moves in the negative direction), so only the positive energy wave packet (particle) impinges on the barrier [28].

\section{Dirac equation wave packets}

A similar construction can be used to build wave packets evolving according to the Dirac equation, starting from an initial state $|G(t=0)\rangle$ of the same form as Eq. (8) now expanded over the Dirac equation plane-wave solutions. The time-evolved wave packet is

$$
\begin{aligned}
G(t, x)= & \sum_{j} \theta_{j} \int d p_{1}\left[c_{D}^{+}\left(p_{1}\right) \Phi_{j}^{+}\left(t, x ; p_{1}\right)\right. \\
& \left.+c_{D}^{-}\left(p_{1}\right) \Phi_{j}^{-}\left(t, x ; p_{1}\right)\right],
\end{aligned}
$$

where the coefficients $c_{D}^{ \pm}=\left\langle\Phi_{1}^{ \pm} \mid G\right\rangle$ are given by Eq. (S-28) of [18].

\section{B. Numerical solutions}

We have computed numerical solutions to the KleinGordon and Dirac equations. This was done by discretizing the corresponding evolution operator in real space for small time steps. The initial wave function $G(t=0, x)$ is discretized on a fixed space grid and the derivatives in the evolution operator are approximated by finite differences in the fourthor fifth-order approximation. The computational details are given in Sec. S4 of [18] (see also Refs. [30-32]).
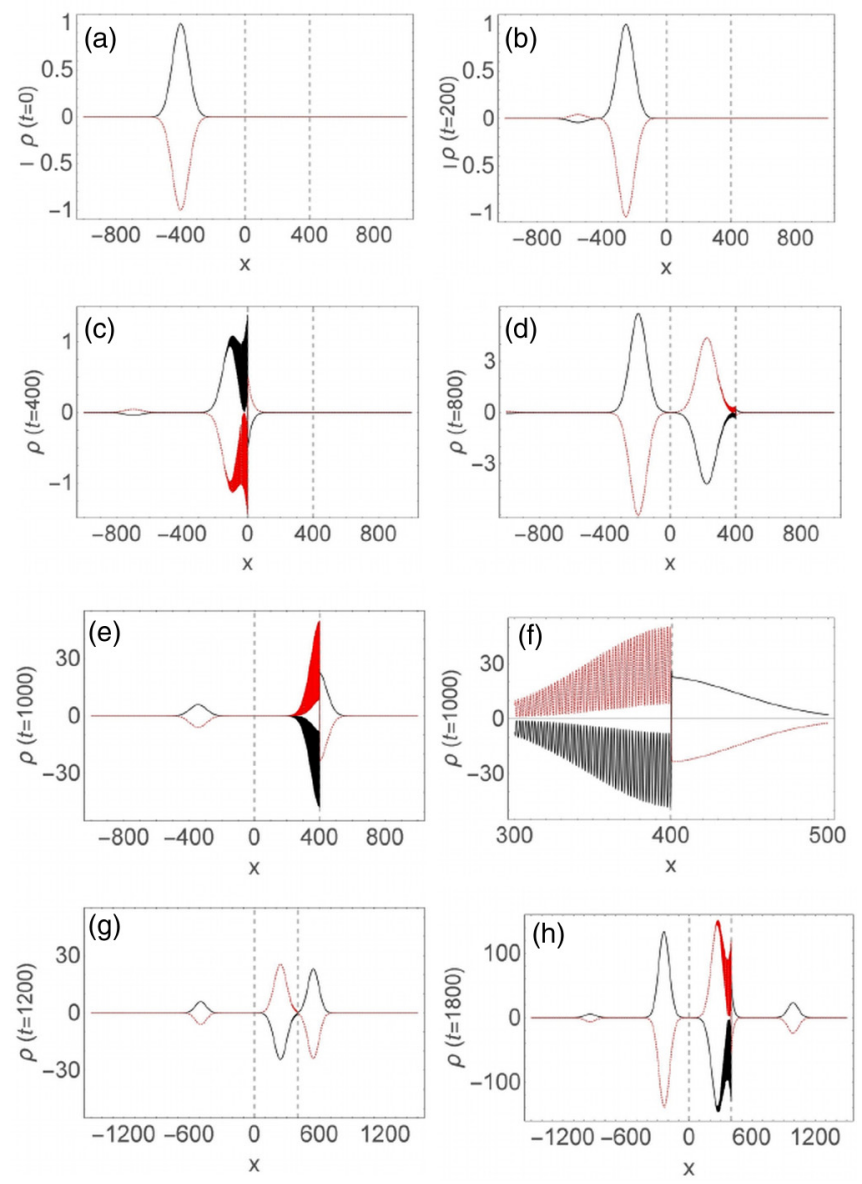

FIG. 2. Wave-packet dynamics for a spin-0 boson described by the Klein-Gordon equation impinging on a smooth supercritical barrier. The charge density $\rho(t)$ is given for different times as specified on each plot. Our semianalytic wave-packet approach is shown in black solid lines, while for the sake of comparison our finitedifference solutions are shown upside-down with a dashed (online: red) line. The supercritical barrier lies within the dotted vertical gray lines. The same initial state, shown in (a), is taken for the wave packet and the numerical calculations and no adjustments or renormalizations are made at longer times (the change in scale reflects charge creation). Note that (f) is a zoom of (e) in the region around the right edge of the barrier. The values of the parameters employed are given in the main text. Natural units ( $\left.\hbar=c=m=\varepsilon_{0}=1\right)$ are used [29].

\section{Illustrative results}

We will now illustrate our wave-packet approach and compare it with fully numerical solutions obtained by solving the relativistic wave equations with a finite-difference scheme. Figure 2 illustrates the wave-packet dynamics for a spin-0 boson impinging on a smooth barrier $V_{s}(x, \epsilon)$ with $\epsilon=5, L=$ 400 , and $V=3.4$ (we use natural units [29]). We pick the initial state, Eq. (8), with $x_{0}=-400, p_{0}=\sqrt{5} / 2$, and $d=50$ (in order to have a rather narrow momentum distribution). We also provide numerical solutions obtained by using our finite-difference scheme introduced above (these solutions are plotted upside-down). The wave packet moves towards the barrier (save for an antiparticle component moving to the left, visible at $t=200$ ), and has appreciably hit the barrier 

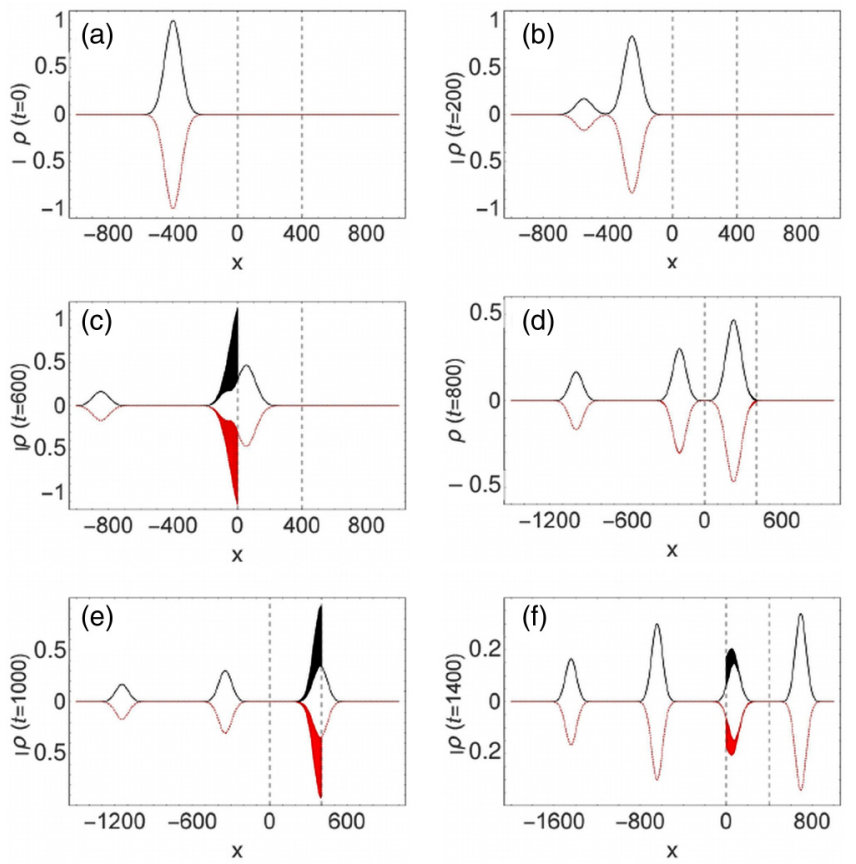

FIG. 3. Wave-packet dynamics for a spin- $1 / 2$ fermion described by the Dirac equation impinging on a supercritical barrier. The density $\rho(t)$ is given for different times as specified. The semianalytic wave-packet approach gives the results shown in black solid lines, while the finite-difference solutions are shown upside-down with a dashed (online: red) line. The supercritical barrier lies within the dotted vertical gray lines. The same initial state, shown in (a), is taken for the wave packet and the numerical calculations and no adjustments or renormalizations are made at longer times. The values of the parameters employed are given in the main text. Natural units ( $\hbar=m=c=\varepsilon_{0}=1$, where $m$ is the electron mass) are used.

by $t=400$, while at $t=800$ one sees Klein tunneling accompanied by charge production both inside and outside the left edge of the barrier (note the vertical scale). At $t=1000$, the antiparticle wave packet hits the right edge of the barrier, inducing additional charge production both for the transmitted (particle) wave packet and for the reflected (antiparticle) one. This motion continues, with the amplitude inside the barrier growing at each reflection.

The MSE-based wave-packet dynamics match very well the computations obtained from the finite-difference solutions. In practice, the number of terms that need to be taken into account in the MSE sum (2) is congruent with the time $t$ at which the wave packet is computed. Indeed, the $n$th term corresponds formally to a wave packet translated by the order of $2 n \pi L$, that for values of $n$ that are high enough did not have time to reach the barrier. Hence these terms do not contribute to the wave packet (in the calculations shown in Fig. 2, including terms up to $n=4$ is sufficient).

The wave-packet dynamics for a spin-1/2 fermion is shown in Fig. 3. We picked the same barrier and initial state parameters as in the Klein-Gordon case except that the smoothness parameter was taken to be the size of the space integration step in the numerical code $(\epsilon \simeq 250)$, effectively corresponding to the rectangular barrier limit. We note again the very good agreement between the wave packets constructed with Eq. (9) and the numerical solutions.

\section{DISCUSSION}

\section{A. General remarks}

We have developed a wave-packet approach to describe Klein tunneling across a supercritical barrier both for KleinGordon and Dirac particles.

The main ingredient was seen to be the multiple scattering expansion, that diverges in the $\mathrm{KG}$ case but converges in the Dirac case.

The main issue now is whether these results lend to a consistent interpretation of the Klein paradox within the first-quantized formalism. This is known to be problematic when basing considerations on stationary plane waves, and indeed different, sometimes conflicting interpretations of superradiance and supercritical tunneling in a first-quantized framework have been given [5-15]. Klein tunneling has even been denied to exist in the bosonic case [14] or for fermions [10]. Of course since particle creation is induced by supercritical potentials, a proper approach requires a QFT treatment. But time-dependent QFT approaches to tackle this problem are scarce (with the exception of the work reviewed in Ref. [23], where supercriticial steps, rather than barriers, were investigated both for fermions and bosons; see also [33], and references therein for recent ramifications of this time-dependent QFT method). Hence, although time-independent approaches to Klein tunneling within the first-quantized framework might be seen as unreliable, it would be worthwhile to consider the merits of a time-dependent first-quantized account, and examine its consistency with time-dependent QFT approaches.

\section{B. Klein paradox in a first-quantized framework}

\section{Bosons}

For the Klein-Gordon equation, the account seems rather simple: A supercritical potential creates positive and negative charges in equal amounts but in different spatial regions. In our wave-packet approach, this is explained by the divergent character of the multiple scattering expansion, although this property is encapsulated in the pseudounitary evolution operator that is solved numerically in the finite-difference code.

Hence, when a particle impinges on a supercritical barrier, the reflected wave packet has a higher amplitude than the incoming one. Similarly, when the Klein tunneling wave packet reaches the right edge of the barrier, a positively charged wave packet leaves the barrier, and this is compensated by additional negative charge inside the barrier. This process goes on indefinitely with positive and negative charge increasing at each reflection (to the extent that the supercritical potential can be maintained despite the growing charge inside the barrier).

It is difficult to accommodate the charge creation mechanism by supercritical fields with the idea that the firstquantized framework would describe a single particle in a superposition of states of different charges (rather than explaining this feature in terms of particle-antiparticle creation). However, leaving this important physical issue aside, we see that the time-dependent wave packets by themselves do account for the bosonic Klein paradox. 


\section{Fermions}

For the Dirac equation, the dynamics is very different. Assume the incoming particle is an electron (with negative charge). Part of the incoming wave packet gets reflected by the barrier, and part gets transmitted. Contrary to the bosonic case, there is no charge creation, so the reflected wave packet has a smaller amplitude than the incoming one. Since the total probability density is conserved, requiring charge conservation implies that the wave-packet tunneling inside the barrier also has negative charge. This is difficult to accommodate within a single-particle picture, even by relying on hole theory.

Indeed, according to the standard hole theory account of the Klein paradox for fermions (see Chap. 12 of [1]), the energy levels of the Dirac sea are raised inside the barrier by the supercritical potential. The incoming electron can then "knock off" an electron from the Dirac sea inside the barrier. This would account for the reflected electron, and would leave a hole in the Dirac sea, corresponding to a positively charged positron. However, we have seen that the wave function we have obtained inside the barrier has an overall negative charge, so that in addition to the hole, an electron should also propagate inside the barrier.

\section{Relation to time-dependent QFT}

Klein tunneling and the Klein paradox ultimately depend on a multiparticle process involving particle-antiparticle pairs creation, that should therefore be described by QFT. A time-dependent QFT scheme [23] has investigated Klein tunneling for rectangular and smooth steps. The QFT calculations have been compared to numerical solutions of the Klein-Gordon [34] and Dirac [19,35] equations.

\section{Bosonic QFT}

In the absence of any incoming particle, space-time dependent charge densities obtained from the bosonic field operator indicate that the supercritical step induces pair creation, particles with positive energies moving away from the step, and wave packets with negative energies (antiparticles) "tunneling" inside the step [34]. When a particle is sent towards the barrier, the QFT computations show that pair creation is enhanced [precisely by the transmission amplitude $t_{l}^{i}$ of Eq. (2)]. This enhancement corresponds to the first-quantized computations for the step. The same correspondence between QFT and our results can be thought to hold in the barrier case: The wave packets we obtained would correspond to the pair creation enhancement produced by sending a particle on the barrier, on top of the pair creation process out of the vacuum. Note that in the barrier case, spontaneous pair creations are also expected to lead to an amplification mechanism through multiple reflections inside the barrier.

\section{Fermionic QFT}

In the Dirac case, QFT computations of the time-dependent spatial densities from the fermionic operators for a step show that an incoming electron modifies the pair production process induced by the supercritical field [35]. The reflected fraction of the incoming first-quantized wave packet appears as an excess of the particle charge (relative to the charge produced by the step). The transmitted wave packet, propagating inside the step, appears instead as a dip in the antiparticle (positronic) charge produced by the supercritical potential. The interpretation is that, as a result of the Pauli principle, the incoming electron decreases the pair-production process that takes place at the step. The decrease of antiparticle production at the edge of the step gives rise to a hole in the positronic charge inside the step. The dip in the electron production is partially compensated by the incoming electron that is interpreted [35] as being fully reflected, yielding overall an excess of electronic charge which is the reflected first-quantized wave packet.

It is not obvious whether one can extrapolate straightforwardly the QFT results for a step to a fermionic barrier. Now both edges of the barrier have a pair-production process. Inside the barrier, the Pauli principle also applies to positrons. This has no counterpart in a first-quantized framework. Nevertheless, one can speculate that, at least for a sufficiently wide barrier, an incoming electron partially suppresses pair production (similarly to the step) at the left edge of the barrier. The hole in positronic charge propagating inside the barrier partially lifts the blockade due to the Pauli principle. Upon reaching the right edge of the barrier, this results in an enhancement in pair production. The wave packet coming out from the barrier in our first-quantized calculations should therefore correspond to the additional electrons produced on the right side of the barrier as the hole reaches the right edge. The additional positrons that are produced are responsible for the smaller amplitude of the hole reflected inside the barrier. This process continues inside the barrier with the hole oscillating with decreasing amplitude. While this QFT-based picture, hinging on the results obtained for a step appear to be consistent with the present first-quantized results, timedependent QFT computations for the barrier would be needed in order to confirm the precise relationship between both pictures of supercritical Klein tunneling.

\section{CONCLUSION}

We have investigated in this paper the Klein paradox for barriers from a time-dependent perspective within the firstquantized framework. We have developed a semianalytical wave-packet approach relying on the properties of a multiple scattering process inside the barrier. This yields a very different behavior for bosons and fermions. In the bosonic case, each collision of the wave packet on an edge of the supercritical potential creates charge (as the multiple scattering process diverges), leading to superradiance. In the Dirac case, Klein tunneling occurs without superradiance (the MSE converges). The wave-packet calculations were complemented with exact numerical solutions obtained by implementing a finite-difference code, leading to an excellent agreement for rectangular and smooth barriers.

We have argued that while a stationary first-quantized approach to the Klein paradox has resulted in different and conflicting interpretations, a time-dependent account adequately describes the dynamics. We further believe such wave-packet calculations might be valuable in order to have a qualitative or quantitative understanding for processes that should in principle be described by space-time QFT approaches, which are computationally much more involved. 


\section{ACKNOWLEDGMENTS}

We would like to thank Rainer Grobe and Charles Su (Illinois State University) for helpful discussions. Financial support of MCIU, through Grant No. PGC2018-101355-
B-100(MCIU/AEI/FEDER,UE) (X.G.d.C., M.P., D.S.), of Spanish MINECO, Project No. FIS2016-80681-P (M.P.), and of the Basque Government Grant No. IT986-16 (M.P., D.S.) is gratefully acknowledged.
[1] W. Greiner, Relativistic Quantum Mechanics (Springer, Berlin, 1996).

[2] J. D. Bjorken and S. D. Drell, Relativistic Quantum Mechanics (McGraw-Hill, New York, 1965).

[3] P. Strange, Relativistic Quantum Mechanics (Cambridge University Press, Cambridge, 1998).

[4] A. Wachter, Relativistic Quantum Mechanics (Springer, Berlin, 2011).

[5] W. Zawadzki and P. Pfeffer, Acta Phys. Pol. B 51, 995 (2020).

[6] K. Kim, Results Phys. 12, 1391 (2019).

[7] I. Morales, B. Neves, Z. Oporto, and O. Piguet, Eur. Phys. J. C 79, 1014 (2019).

[8] D. Xu, T. Wang, and X. Xue, Found Phys. 43, 1257 (2013).

[9] A. D. Alhaidari, Phys. Scr. 83, 025001 (2011).

[10] Y. V. Kononets, Found Phys. 40, 545 (2010).

[11] S. D. Bosanac, J. Phys. A 40, 8991 (2007).

[12] P. Christillin and E. d'Emilio, Phys. Rev. A 76, 042104 (2007).

[13] S. De Leo and P. P. Rotelli, Phys. Rev. A 73, 042107 (2006).

[14] P. Ghose, M. K. Samal, and A. Datta, Phy. Lett. A 315, 23 (2003).

[15] H. Nitta and T. Kudo, and H. Minowa, Am. J. Phys. 67, 966 (1999).

[16] F. Nieto-Guadarrama and J. Villavicencio, Phys. Rev. A 101, 042104 (2020).

[17] S. Colin and A. Matzkin, Europhys. Lett. 130, 50003 (2020).

[18] See Supplemental Material at http://link.aps.org/supplemental/ 10.1103/PhysRevA.103.042203 for details on the derivation of the MSE amplitudes, wave packets construction, and the numerical method employed.

[19] P. Kennedy, J. Phys. A: Math. Gen. 35, 689 (2002); C. Rojas, Can. J. Phys. 93, 85 (2014).
[20] C. A. Manogue, Ann. Phys. 181, 261 (1988).

[21] N. Dombey and A. Calogeracos, Phys. Rep. 315, 41 (1999).

[22] A. Hansen and F. Ravndal, Phys. Scr. 23, 1036 (1981).

[23] T. Cheng, Q. Su, and R. Grobe, Contemp. Phys. 51, 315 (2010).

[24] S. P. Gavrilov and D. M. Gitman, Phys. Rev. D 93, 045002 (2016).

[25] X. Gutierrez de la Cal, M. Alkhateeb, M. Pons, A. Matzkin, and D. Sokolovski, Sci. Rep. 10, 19225 (2020).

[26] M. I. Katsnelson, K. S. Novoselov, and A. K. Geim, Nat. Phys. 2, 620 (2006).

[27] R.-K. Suff, G. G. Siu, and X. Chou, J. Phys. A: Math. Gen. 26, 1001 (1993).

[28] We might as well have chosen to restrict $G(0, x)$ to the expansion over the positive energy eigenstates in Eq. (7), but when comparing with numerical solutions it is more interesting to keep (8) as the initial state.

[29] We use natural units $\hbar=m=c=\varepsilon_{0}=1$ where $\varepsilon_{0}$ is the vacuum permittivity. For the Dirac equation, $m$ is the electron mass. For the Klein-Gordon equation, the conversion to SI units depends on the specific boson mass $m$, e.g., for a pion meson $\pi^{+}$ the mass entering the conversion factor is $139.57 \mathrm{MeV} / \mathrm{c}^{2}$.

[30] M. Ruf, H. Bauke, and C. H. Keitel, J. Comput. Phys. 228, 9092 (2009).

[31] F. Blumenthal and H. Bauke, J. Comput. Phys. 231, 454 (2012).

[32] C. Mueller, N. Gruen, and W. Scheid, Phys. Lett. A 242, 245 (1998).

[33] Y. Lu, N. Christensen, Q. Su, and R. Grobe, Phys. Rev. A 101, 022503 (2020).

[34] R. E. Wagner, M. R. Ware, Q. Su, and R. Grobe, Phys. Rev. A 81, 024101 (2010).

[35] P. Krekora, Q. Su, and R. Grobe, Phys. Rev. A 72, 064103 (2005). 